\title{
Ethical neuroscience
}

\author{
Although institutional review boards are important ethical gatekeepers of human patient research, there is little \\ data to evaluate their effectiveness. More coordination and a more transparent decision-making process is critical if \\ review boards are to make appropriate and consistent decisions.
}

A $\mathrm{n}$ ethical overview is meant to be more than just another bureaucratic hurdle in doing research; it is a guarantee that all research is held to certain minimum standards and, particularly for human patient research, it is an assurance that the participants' welfare is being looked after and that the risk to them is minimized. However, there is very little oversight of how well this overview meets its stated aims, especially for human research. Moreover, what little data exists points to some worrying inconsistencies; a study that submitted a mock functional magnetic resonance imaging human neuroimaging protocol to 43 Canadian review ethics boards found that the protocol was unconditionally approved by 3 boards, approved conditionally by 10 and rejected by 30 (ref. 1). Given the increasingly knotty ethical challenges that neuroscience advances present, it is critical that we try to improve this situation by encouraging review boards to make their decision-making process more open and by encouraging greater crosstalk between different ethical review boards.

All experiments in humans have to abide by the general 1947 Nuremberg code guidelines. These stipulate obtaining voluntary, informed consent, avoiding unnecessary suffering and that studies have to be based on prior animal work. However, how these broad guidelines are implemented differs widely. In France, according to Erwan Bezard from the University of Bordeaux, the consensus is that invasive scientific investigations inside the human brain should be avoided unless there is a clear therapeutic benefit to the patient. Interestingly, however, when studies performed elsewhere have demonstrated clear therapeutic benefit, these restrictions may be relaxed to allow follow-up investigations. Although other countries partially hold to the same standard, in that any invasive investigations (such as electrophysiological recordings) happen only when therapeutic interventions present the opportunity, there are many studies (for example, see ref. 2) that do adhere to the standards set by their institution, but involve interventions that appear to have no direct benefit to patients.

Standards also differ on how much human studies need to be supported by previous work in animals, which provides an idea of the risks involved. This is further complicated by the fact that there are no universally accepted models for many diseases and processes. These factors mean that it is impossible to offer very specific, universally binding guidelines, making the job of institutional review boards all the more difficult. These decisions also increasingly require more specialist knowledge, as increasingly more esoteric techniques allow for more complicated experiments. The standards in specific fields are thus always evolving, but it is difficult for the average researcher (who may not even be a neuroscientist) on an institutional review board to be cognizant of all of the issues involved.

One way to help come up with some community standards in this rapidly evolving field may be to create a publicly available, searchable database along the lines of the existing US clinical trials database (http://clinicaltrials.gov). This would also provide a more systematic way of highlighting decisions that differ from the norm and may be useful for providing guidelines for individual review boards.

The other strategy for ensuring greater consistency is to promote greater cross-talk between local institutional review boards. This kind of exchange would provide a chance to develop consensus on ethical issues raised by common study protocols and to share expertise and to articulate questions that should be asked for some studies. Such discussion is also useful in providing lists of relevant outside experts who could comment on especially difficult decisions or unusual study protocols. Although there are occasional meetings that encourage such discussion, their frequency and attendance varies across different countries; for example, in France, all of the local Comité pour la Protection des Personnes (committees for the protection of individuals, which fulfill the same function as institutional review boards elsewhere) are required to attend an annual national meeting. In other countries, requirements are less formalized.

Potentially, greater consistency would also result if the ethical decisionmaking process was more centralized, but such attempts have yielded mixed results. In theory, centralization should facilitate increased expertise by pooling resources and reducing costs. In practice, it is not clear that centralization makes the decision making more informed or efficient. For instance, although the UK has had a Central Office for Research Ethics Committees since 2007, most studies in the UK still have to seek approval from individual research departments. Indeed, a 2009 study (http://jrsm. rsmjournals.com/cgi/reprint/102/5/195.pdf) reported that approval for a multicenter, non-interventional study could take as long as 147 days, and claims that the perceived difficulties in obtaining ethical approval in the UK have discouraged multinational studies from involving a UK center.

Given the very valuable data that human patient studies provide, it is critical that we take steps to improve the ethical review process and that we ensure that the institutional review boards have the resources to make fully informed decisions. What is urgently needed is some real data on how the current process is working. Providing a searchable database of current protocols of the sort already provided for clinical studies would be a good first step by providing guidance to local review boards about decisions made on comparable cases, while still retaining the flexibility required to make case-by-case decisions. It would also highlight decisions that differ from the norm. Along with greater cross-talk between local ethical review boards, such publicly available information would also help reassure the public that ethical review is indeed doing what it sets out to do, by ensuring the welfare of subjects while advancing our knowledge of how the human brain works.

1. de Champlain, J. \& Patenaude, J. J. Med. Ethics 32, 530-534 (2006).

2. Fedele, E. et al. Exp. Neurol. 167, 356-365 (2001). 